\title{
Diagnostic utility of SOX10 to distinguish malignant peripheral nerve sheath tumor from synovial sarcoma, including intraneural synovial sarcoma
}

\author{
Yuna Kang ${ }^{1}$, Melike Pekmezci ${ }^{1}$, Andrew L Folpe ${ }^{2}$, Ayca Ersen ${ }^{2}$ and Andrew E Horvai ${ }^{1}$ \\ ${ }^{1}$ Department of Pathology, University of California, San Francisco, CA, USA and ${ }^{2}$ Department of Laboratory \\ Medicine and Pathology, Mayo Clinic, Rochester, MN, USA
}

\begin{abstract}
Synovial sarcoma and malignant peripheral nerve sheath tumor pose a significant diagnostic challenge given similar histomorphology. The distinction is further complicated by similar immunophenotype and especially by occasional synovial sarcomas that present as intraneural tumors. Although the presence of a $t(X ; 18)$ rearrangement or expression of TLE1 can help confirm the diagnosis of synovial sarcoma, negative results for these tests are not diagnostic of malignant peripheral nerve sheath tumor. The SOX10 transcription factor, a putative marker of neural crest differentiation, may have diagnostic utility in this differential, but immunohistochemical data are limited. The goal of the present study was to determine the diagnostic utility of SOX10 to discriminate between synovial sarcoma and malignant peripheral nerve sheath tumor. Forty-eight cases of malignant peripheral nerve sheath tumor, all from patients with documented neurofibromatosis, and 97 cases of genetically confirmed synovial sarcoma, including 4 intraneural synovial sarcomas, were immunohistochemically stained for SOX10. The stain was scored for intensity and fraction of cells staining. Thirty-two of 48 malignant peripheral nerve sheath tumors $(67 \%)$ were SOX10-positive. The majority of malignant peripheral nerve sheath tumors showed $\geq 2+$ staining, but staining did not correlate with grade. By contrast, only $7 / 97(7 \%)$ synovial sarcomas were SOX10-positive. Only three synovial sarcomas showed $\geq 2+$ staining but, importantly, two of these were intraneural synovial sarcoma. Therefore, SOX10 is a specific (93\%), albeit not very sensitive $(67 \%)$, diagnostic marker to support a diagnosis of malignant peripheral nerve sheath tumor over synovial sarcoma. Furthermore, the stain needs to be interpreted with caution in intraneural tumors in order to avoid a potential diagnostic pitfall. It remains to be determined whether SoX10-positive cells in intraneural synovial sarcoma represent entrapped Schwann cells, synovial sarcoma cells or both.
\end{abstract}

Modern Pathology (2014) 27, 55-61; doi:10.1038/modpathol.2013.115; published online 9 August 2013

Keywords: malignant peripheral nerve sheath tumor; SOX10; synovial sarcoma

Malignant peripheral nerve sheath tumors are malignant neoplasms that arise from peripheral nerves or extraneural soft tissue, demonstrating differentiation along the lines of peripheral nerve sheath elements. ${ }^{1-3}$ The classic histological appearance of malignant peripheral nerve sheath tumor is that of tightly packed fascicles composed of spindled-to-fusiform cells with hyperchromatic nuclei and indistinct cytoplasm. ${ }^{2}$ Heterologous

Correspondence: Dr AE Horvai, MD, PhD, Department of Pathology, University of California, 1600 Divisadero Drive B220, San Francisco, CA 94115-1656, USA.

E-mail: andrew.horvai@ucsf.edu

Received 20 March 2013; revised 15 May 2013; accepted 15 May 2013; published online 9 August 2013 differentiation, including gland formation, can be present in rare cases. ${ }^{4}$ Despite advances in the immunohistochemical and molecular diagnosis of sarcomas, the diagnosis of malignant peripheral nerve sheath tumor remains problematic. In general, this diagnosis relies on (1) the contiguity with a benign nerve sheath tumor, a prominent feature typically seen in malignant peripheral nerve sheath tumor arising in patients with neurofibromatosis type 1 (NF1), or (2) major nerve involvement and (3) exclusion of other spindle cell lesions. ${ }^{5}$ In most situations, synovial sarcoma is among the closest morphological mimics of malignant peripheral nerve sheath tumor that must be ruled out. ${ }^{4}$

Synovial sarcoma is a malignant mesenchymal neoplasm of unknown histotype, characterized by a 
balanced reciprocal translocation $\mathrm{t}(\mathrm{X} ; 18)$ and usually at least focal evidence of epithelial differentiation. ${ }^{6-8}$ Histologically, synovial sarcoma is divided into three subtypes: ${ }^{9}$ Monophasic synovial sarcoma is composed solely of uniform spindled cells, while biphasic synovial sarcoma shows glandular differentiation. The third, or poorly differentiated, subtype consists of primitive round cells or larger anaplastic cells with elevated mitotic activity and necrosis.

The distinction between synovial sarcoma and malignant peripheral nerve sheath tumor presents a diagnostic challenge because of morphological, immunophenotypical and clinical overlap. In addition to the morphological overlap between malignant peripheral nerve sheath tumor and the more common monophasic synovial sarcoma, poorly differentiated forms of both tumors are quite similar. Although malignant peripheral nerve sheath tumor with glandular differentiation is admittedly rare, it can be confused with biphasic synovial sarcoma. S100 protein, a marker of nerve sheath differentiation has limited diagnostic utility as higher grade malignant peripheral nerve sheath tumors are often negative ${ }^{1}$ and up to a third of synovial sarcomas are positive. ${ }^{10}$ The clinical presentation of both tumors, namely a large soft tissue mass with a predilection for the extremities of young-to-middle-aged adults, is also quite similar. ${ }^{5,11-13}$ The existence of intraneural examples of synovial sarcoma blurs the clinical distinction further. ${ }^{14}$ Therefore, the clinical history of NF1 is often the 'gold standard' to establish the diagnosis of malignant peripheral nerve sheath tumor. Germline mutations of the neurofibromin 1 tumor-suppressor gene increase the risk of malignant peripheral nerve sheath tumor and tumors present at a younger age than sporadic cases. ${ }^{5,15}$ Although NF1 patients are at greater risk for malignancies other than malignant peripheral nerve sheath tumor than the general population, the incidence of synovial sarcoma does not appear to be increased. ${ }^{16}$

Following the discovery of the synovial sarcomaspecific translocation $\mathrm{t}(\mathrm{X} ; 18),{ }^{17}$ molecular testing has been employed as a reliable diagnostic method in making the distinction between synovial sarcoma and other histological mimics. The translocation results in the fusion of $S S 18$ to the $S S X 1, S S X 2$ or SSX 4 gene. ${ }^{8}$ Despite the specificity of the molecular findings of synovial sarcoma, immunohistochemical testing remains more widely available to most pathology practices. Malignant peripheral nerve sheath tumors appear to be a more genetically diverse family of sarcomas, and no reproducible genetic or molecular test is currently in routine use.

SOX10 (SRY-related HMG box 10) was recently identified as a transcription factor implicated in neural crest differentiation. ${ }^{18,19}$ Previous studies have shown that the expression of this nuclear transcription factor, detected by immunohistochemistry, is sensitive for a variety of Schwannian and melanocytic neoplasms, ${ }^{20-22}$ benign myoepithelial cells and rarely other tumor types. ${ }^{23}$ However, data specifically comparing genetically confirmed synovial sarcoma, including intraneural synovial sarcoma, with malignant peripheral nerve sheath tumor with respect to SOX10 expression is limited. In the present study, we evaluate the utility of SOX10 immunohistochemistry in differentiating malignant peripheral nerve sheath tumor from synovial sarcoma.

\section{Materials and methods}

\section{Selection of Cases}

The pathology archives of the University of California San Francisco and Mayo Clinic were searched for the diagnoses of synovial sarcoma and malignant peripheral nerve sheath tumor. Clinical data were retrieved for all cases and H\&E sections were reviewed. Only those synovial sarcomas with confirmation of $S S 18$ rearrangement by fluorescence in situ hybridization or cytogenetics or SS18-SSX fusion by RT-PCR were included. Only those malignant peripheral nerve sheath tumors from patients with a history of NF1 were included. Furthermore, regardless of NF1 clinical status, cutaneous tumors for which melanoma was a consideration or nerve sheath tumors with equivocal features of malignancy were excluded. Subsets of these malignant peripheral nerve sheath tumor and synovial sarcoma cases have been previously published. ${ }^{24,25}$

\section{Grading of Malignant Peripheral Nerve Sheath Tumor}

Although controversy exists regarding the value of grading of malignant peripheral nerve sheath tumors for prognostic purposes, ${ }^{5,26}$ we aimed to test for the association between histological grade and SOX10 expression. Consequently, malignant peripheral nerve sheath tumor was stratified into low and high grade forms based on the criteria of Ducatman et al. ${ }^{5}$

\section{Immunohistochemistry}

Immunohistochemical analysis was performed on archival, paraffin-embedded sections. Whole slide sections were used for all malignant peripheral nerve sheath tumor cases and 45 of 97 synovial sarcoma cases. The remaining 52 synovial sarcoma cases were analyzed in triplicate on a previously constructed tissue microarray ${ }^{25}$ because of financial constraints and because paraffin blocks were not available for all synovial sarcoma cases. Four- $\mu \mathrm{m}$ paraffin sections were stained using standard techniques. Slides were subjected to antigen recovery with $10 \mathrm{mM}$ citrate buffer in a pressure cooker, then incubated with the SOX10 goat polyclonal antibody (sc-17342, Santa Cruz 
Biotechnology, Santa Cruz, CA, USA) at 1:50 dilution. Detection was performed using the ABC detection method (Vector Labs, Burlingame, CA, USA), according to the manufacturer's directions.

Nuclear staining was evaluated according to the four-point system of Adams et $a l^{27,28}$ as follows:

- Strong $(3+)$ : dark staining that is easily visible at low power and involves $>50 \%$ of cells.

- Moderate $(2+)$ : focal darkly staining areas $(<50 \%$ of cells) or moderate staining of $>50 \%$ of cells.

- Weak $(1+)$ : focal moderate staining in $<50 \%$ of cells or pale staining in any proportion of cells not easily seen at low power.

- Negative $(0+)$ : None of the above.

The presence of neural tissue in the intraneural synovial sarcomas was based on H\&E morphology and confirmed by immunostaining using the neurofilament monoclonal antibody (2F11, Cell Marque, Rocklin, CA, USA) at full concentration. Antigen recovery and detection were performed as described above.

\section{Results}

Patients with NF1-associated malignant peripheral nerve sheath tumor consisted of 26 males and 22 females. The average age at diagnosis was 32 years (range 11-86). Forty-one of 48 cases (85\%) were high grade.

Results of immunohistochemistry for malignant peripheral nerve sheath tumor are summarized in Table 1 and representative images in Figure 1. SOX10 staining showed nuclear positivity in 32 of 48 cases $(67 \%)$ and most of the positive cases ( $n=19,60 \%)$ demonstrated $\geq 2+$ staining. One malignant peripheral nerve sheath tumor with glandular differentiation was identified and showed positive $(2+$ staining, Figures $1 \mathrm{e}$ and $\mathrm{f})$ in the spindle cell component. No statistically significant correlation $(P=0.16$, Fisher's exact test) was noted between SOX10 staining and malignant peripheral nerve sheath tumor grade.

The synovial sarcoma patients included 53 males, 42 females and two in whom the gender was not documented. The average age was 35 years (range
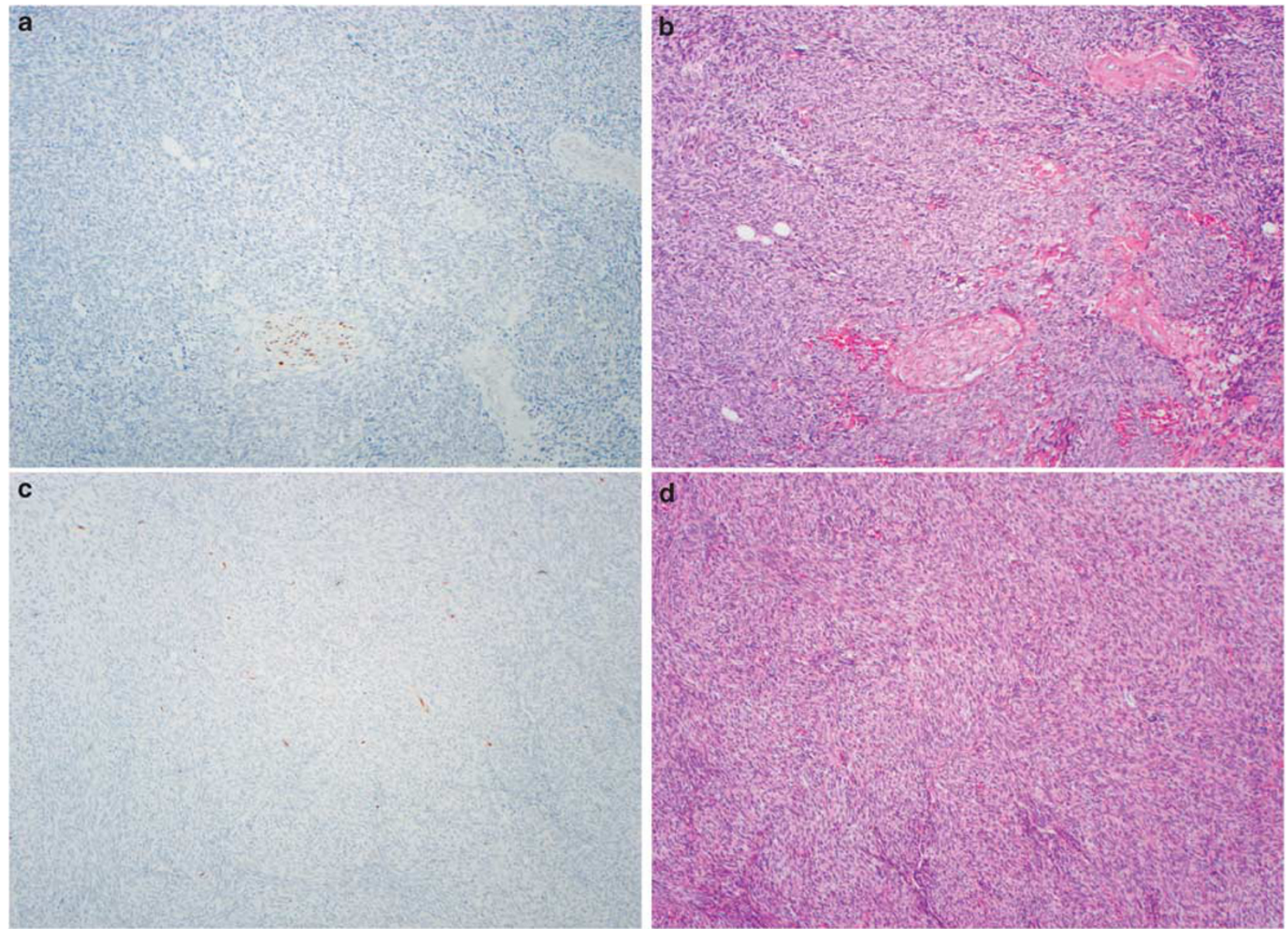

Figure 1 Representative SOX10 immunohistochemical results and corresponding H\&E morphology of malignant peripheral nerve sheath tumor. (a, b) $0+$, negative: No nuclear positivity in the tumor, internal positive control of entrapped nerve; (c, d) $1+$ : moderate staining in $<50 \%$ of cells; (e, f) $2+$, malignant peripheral nerve sheath tumor with glandular differentiation showing focal darkly staining areas in $<50 \%$ of cells, note the absence of nuclear staining in glands. (g, h) $3+$, strong: dark staining that is easily visible at low power and involves $>50 \%$ of cells. 

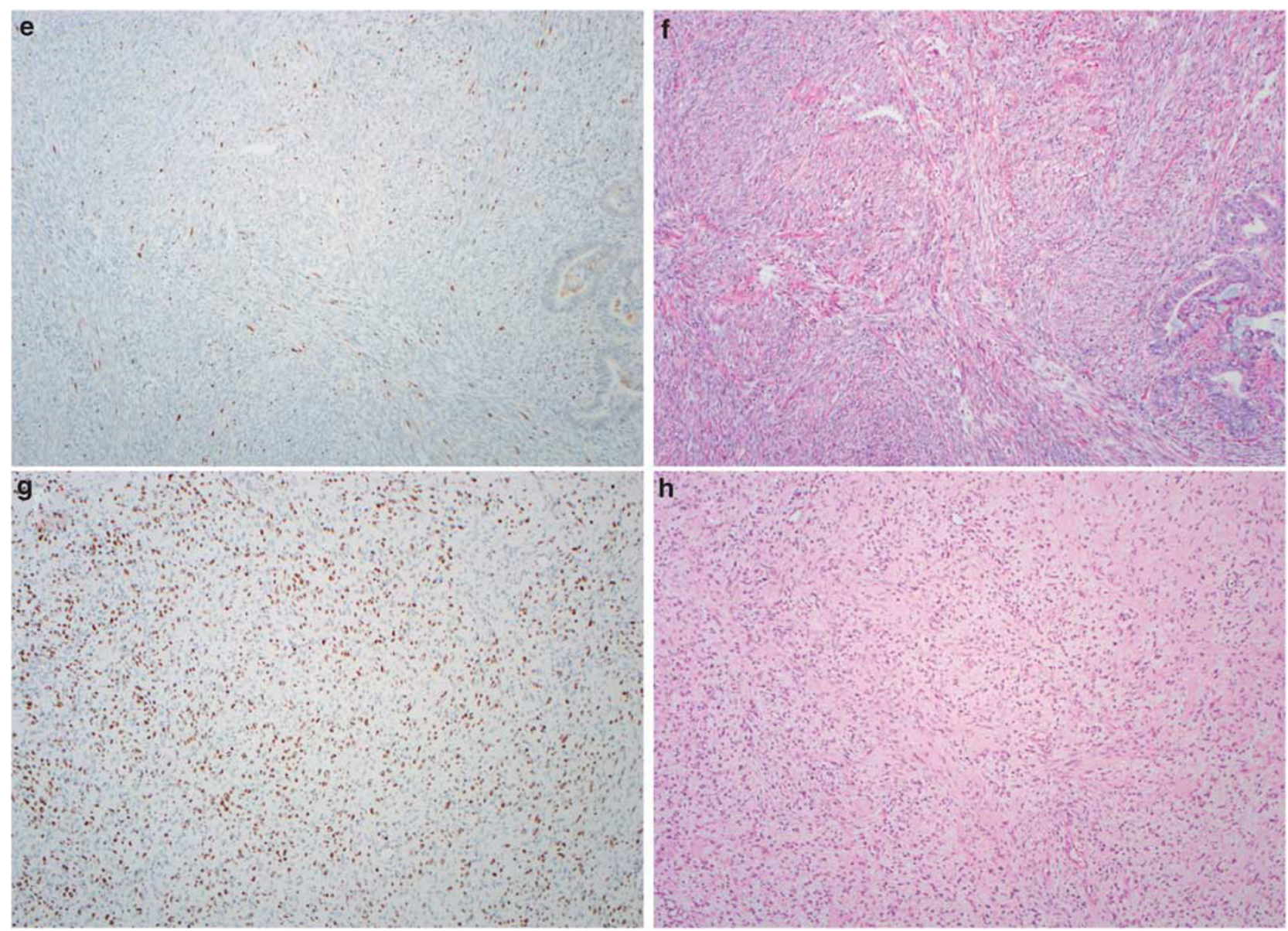

Figure 1 Continued.

8-77). The most common site was the lower extremity $(n=50,52 \%)$. Of the 97 synovial sarcoma cases, $69(71 \%)$ were monophasic, $26(27 \%)$ were biphasic and $2(2 \%)$ were poorly differentiated. Four $(4 \%)$ of the synovial sarcomas were intraneural based on H\&E findings and the presence of neurofilament staining of entrapped axons (Figure 2), all of which were monophasic.

Positive SOX10 staining, summarized in Table 1, was only seen in 7 of $97(7 \%)$ synovial sarcomas, 6 of which were monophasic and 1 was biphasic. Nuclear staining was observed only in the spindled cells of the biphasic case. Of the 7 SOX10-positive cases, most $(n=4,57 \%)$ showed only $1+$ staining. The remaining three positive cases showed $2+$ staining, but, intriguingly, two of these cases were intraneural (Figure 2). The SOX10-positive cells were relatively evenly distributed but were fewer in number than the Schwann cells of the adjacent benign nerve. Although it was difficult to be certain that these positive cells did not represent entrapped Schwann cells, rather than tumor cells, it was decided to regard these cases as 'positive' for the sake of objective analysis. No synovial sarcoma case showed $3+$ staining. There was complete concordance between cores on the synovial sarcoma tissue microarray. The fraction of SOX10-positive cases did not differ significantly $(P=0.27$, Fisher's exact test) between those tested on tissue microarray $(2 / 52,4 \%)$ compared with those tested on whole sections $(5 / 45,11 \%)$.

The sensitivity and specificity of SOX10 to distinguish malignant peripheral nerve sheath tumor from synovial sarcoma were 67 and 93\%, respectively. The positive-predictive and negativepredictive values were 82 and $89 \%$, respectively.

\section{Discussion}

The distinction between malignant peripheral nerve sheath tumor and synovial sarcoma continues to be a challenge to pathologists given their clinical, morphological and immunophenotypical overlap. Both tumors most commonly present as large, deep-seated soft tissue masses composed of highly cellular, monotonous spindle cells in a fascicular growth pattern. Most immunohistochemical markers associated with malignant peripheral nerve sheath tumors, such as bcl2, CD56, nestin and S100 protein, are also expressed in a significant proportion of synovial sarcoma. ${ }^{10,12,29-31}$ TLE1 shows promise as a marker of synovial sarcoma, but weak and variable 

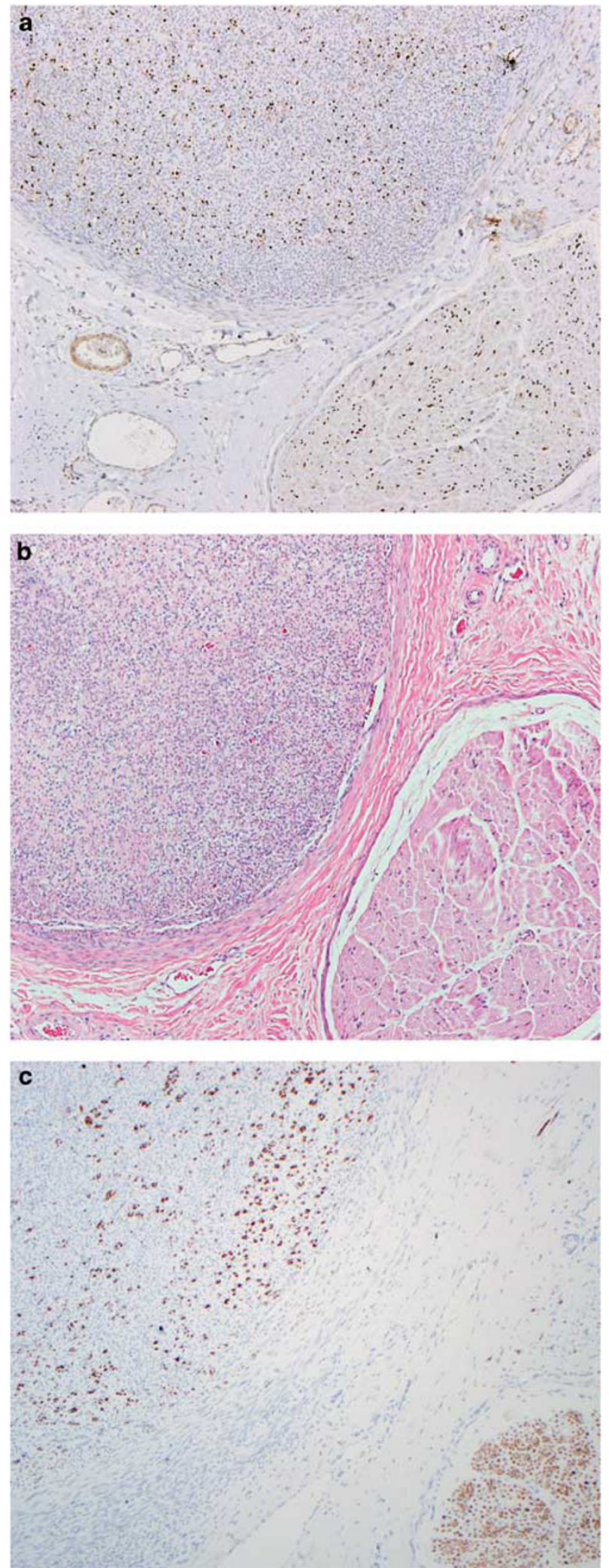

Figure 2 Representative SOX10 expression in intraneural synovial sarcoma. (a) Intraneural synovial sarcoma (left) with $2+$ SOX10 staining. The adjacent nerve (right) serves as an internal positive control. (b) Corresponding H\&E stain. (c) Neurofilament immunostain demonstrating residual neural tissue within synovial sarcoma.
Table 1 Summary of SOX10 immunohistochemical staining results in malignant peripheral nerve sheath tumors and synovial sarcomas

\begin{tabular}{lrrrrr}
\hline & \multicolumn{3}{c}{ SOX10 staining score } & \\
\cline { 2 - 5 } & $0+$ & $1+$ & $2+$ & $3+$ & Total positive \\
\hline MPNST $(n=48)$ & 16 & 13 & 16 & 3 & $32(67 \%)$ \\
SS $(n=97)$ & & & & & \\
$\quad \begin{array}{l}\text { Extraneural }(n=93) \\
\quad \text { Intraneural }(n=4)\end{array}$ & 88 & 4 & 1 & 0 & $5(5 \%)$ \\
\hline
\end{tabular}

MPNST, malignant peripheral nerve sheath tumor; SS, synovial sarcoma.

expression can be seen in malignant peripheral nerve sheath tumors. ${ }^{32-35}$

Consequently, genetic and molecular tests to demonstrate $\mathrm{t}(\mathrm{X} ; 18)$ rearrangement or SS18-SSX fusion, respectively, have become routine at many specialized centers to confirm the diagnosis of synovial sarcoma. Although some controversy existed in the past regarding the specificity of this genetic abnormality, ${ }^{36,37}$ the current consensus among sarcoma pathologists is that SS18-SSX fusions are highly specific for synovial sarcoma. Malignant peripheral nerve sheath tumor is a genetically heterogeneous group of tumors, and the absence of $t(X ; 18)$ in a monomorphic spindle cell neoplasm is certainly not diagnostic of malignant peripheral nerve sheath tumor. Thus, the need exists for a positive confirmatory immunohistochemical test to support the diagnosis of malignant peripheral nerve sheath tumor over synovial sarcoma.

The SOX (SRY-related HMG box) transcription factor family was first discovered in 1990 and was later revealed to have a critical role in the developmental process. ${ }^{38-40}$ The SOX genes are divided into subfamilies primarily based on the amino-acid sequence homology of the HMG domain. ${ }^{41}$ SOX10 is thought to have an essential role in differentiation of neural crest into Schwann cells, astrocytes and oligodendrocytes. ${ }^{40,42,43}$ SOX10 positivity has also been observed in other tissues such as myoepithelial cells of the breast and acinar and myoepithelial cells of the salivary glands.

The diagnostic utility of anti-SOX10 antibodies has been examined primarily in melanocytic and Schwannian tumors. To date, data comparing SOX10 immunostaining in malignant peripheral nerve sheath tumor and genetically confirmed synovial sarcoma are limited. ${ }^{21,22,44}$ The sensitivity of SOX10 for malignant peripheral nerve sheath tumor varies between studies (27-61\%), possibly related to the inclusion criteria for malignant peripheral nerve sheath tumor or differences in antibodies or staining procedures. In the most recent study by Karamchandani et $a l,{ }^{22}$ which compared 78 cases of malignant peripheral nerve sheath tumor 
and 79 synovial sarcoma, (including 61 malignant peripheral nerve sheath tumor and 15 synovial sarcoma previously reported by Nonaka et $a{ }^{21}$ ) SOX10 was found to be more specific for malignant peripheral nerve sheath tumor than was S100 protein.

The results from our study indicate that SOX10 expression has moderate sensitivity $(67 \%)$ and high specificity $(93 \%)$ for malignant peripheral nerve sheath tumor, when synovial sarcoma is in the differential diagnosis. We considered the possibility that the lack of sensitivity of SOX10 may, in a manner analogous to $\mathrm{S} 100$ protein, be related to loss of expression in higher grade tumors. ${ }^{1}$ However, we noted no correlation between malignant peripheral nerve sheath tumor grade and SOX10 expression arguing against this hypothesis. Previous studies evaluating SOX10 expression in malignant peripheral nerve sheath tumor have included a mixed population of both NF1-associated and sporadic cases. ${ }^{22,44}$ Nevertheless, the diagnostic criterion of a spindle cell malignancy associated with nerve, even if focally S100 protein positive, in our opinion does not have sufficient specificity to exclude other possible diagnoses, including melanoma, sarcomatoid carcinoma and, most importantly, intraneural synovial sarcoma. Therefore, a highly selected population of NF1-associated malignant peripheral nerve sheath tumor is the best standard by which to study the diagnostic utility of SOX10. It is reassuring, however, that of 52 probable sporadic malignant peripheral nerve sheath tumors (based on association with a pre-existing benign nerve sheath tumor and the absence of clinical evidence of NF1), $25(48 \%)$ were SOX10-positive (Andrew E Horvai and Andrew L Folpe, unpublished observations).

To our knowledge, the current study is the first to specifically address SOX10 immunohistochemistry in malignant peripheral nerve sheath tumor with glandular differentiation and intraneural synovial sarcoma-morphological patterns that can be especially challenging. These variants are, of course, extremely rare, so that even in a large series the number of cases precludes robust statistical analysis. Nevertheless, our findings suggest that the presence of glandular differentiation in malignant peripheral nerve sheath tumor does not affect SOX10 expression in the spindle cell component. By contrast, two of the four intraneural synovial sarcomas contained a significant number of SOX10-positive cells, of uncertain lineage. These two cases represented two of the three synovial sarcomas with at least $2+$ staining score. Therefore, the SOX10 stain needs to be interpreted with caution when examining intraneural tumors because a positive result, even if strong, does not exclude synovial sarcoma. It remains to be determined whether Sox-10-positive cells in intraneural synovial sarcoma represent entrapped Schwann cells, synovial sarcoma cells or both.

In summary, SOX10 appears to be a moderately sensitive but very specific marker of malignant peripheral nerve sheath tumor, independent of grade. In situations where genetic or molecular testing for $\mathrm{t}(\mathrm{X} ; 18)$ is not available, not informative or yields a negative result, SOX10 immunohistochemistry can be a useful adjunct. A positive result in an intraneural tumor, however, does not exclude the diagnosis of synovial sarcoma and could be a potential diagnostic pitfall.

\section{Acknowledgements}

This research is supported by the Residents' Teaching and Research Endowments from UCSF Department of Pathology. We would like to acknowledge the late Dr Bernd W Scheithauer for assembling such an extensive and thoroughly documented collection of malignant peripheral nerve sheath tumors and the UCSF Comprehensive Cancer Center immunohistochemistry core for their technical assistance.

\section{Disclosure/conflict of interest}

The authors declare no conflict of interest.

\section{References}

1 Scheithauer BW, Louis DN, Hunter S, et al. Malignant peripheral nerve sheath tumor, In: Louis DN, Ohgaki H, Wiestler OD, Cavenee WK (eds) World Health Organization Classification of Tumours of the Central Nervous System, 4th edn. IARC Press: Lyon, France, 2007, pp 160-162.

2 Weiss SW, Goldblum JR. Enzinger and Weiss's Soft Tissue Tumors. Mosby: St. Louis, MO, USA, 2008, pp 903-941.

3 Nielsen GP, Antonescu CR, Lothe RA. Malignant peripheral nerve sheath tumor. In: Fletcher CD, Bridge JA, Hogendoorn PC, Mertens F (eds). WHO Classification of Tumours of Soft Tissue and Bone, 4th edn. vol. (IARC Press: Lyons, France. 2013, pp 187-188.

4 Rodriguez FJ, Folpe AL, Giannini C, et al. Pathology of peripheral nerve sheath tumors: diagnostic overview and update on selected diagnostic problems. Acta Neuropathol 2012;123:295-319.

5 Ducatman BS, Scheithauer BW, Piepgras DG, et al. Malignant peripheral nerve sheath tumors. A clinicopathologic study of 120 cases. Cancer 1986;57: 2006-2021.

6 Turc-Carel C, Dal Cin P, Limon J, et al. Involvement of chromosome $\mathrm{X}$ in primary cytogenetic change in human neoplasia: nonrandom translocation in synovial sarcoma. Proc Natl Acad Sci USA 1987;84: 1981-1985.

7 Sandberg AA, Bridge JA. Updates on the cytogenetics and molecular genetics of bone and soft tissue tumors. Synovial sarcoma. Cancer Genet Cytogenet 2002;133: 1-23.

8 Suurmeijer AJ, De Bruijn DR, Geurts van Kessel A, et al. Synovial sarcoma, In: Fletcher CD, Bridge JA, Hogendoorn PC, Mertens F (eds) WHO Classification of Tumours of Soft Tissue and Bone, 4th edn. IARC Press: Lyon, France, 2013, pp 213-215. 
9 Fisher C. Synovial sarcoma. Ann Diagn Pathol 1998;2: 401-421.

10 Guillou L, Kraus M, Dei Tos AP, et al. S-100 protein reactivity in synovial sarcomas-a potentially frequent diagnostic pitfall. Immunohistochemical analysis of 100 cases. Appl Immunohistochem 1997;4:167-175.

11 Daimaru Y, Hashimoto H, Enjoji M. Malignant peripheral nerve-sheath tumors (malignant schwannomas). An immunohistochemical study of 29 cases. Am J Surg Pathol 1985;9:434-444.

12 Eilber FC, Dry SM. Diagnosis and management of synovial sarcoma. J Surg Oncol 2008;97:314-320.

13 Palmerini E, Staals EL, Alberghini M, et al. Synovial sarcoma: retrospective analysis of 250 patients treated at a single institution. Cancer 2009;115:2988-2998.

14 Scheithauer BW, Amrami KK, Folpe AL, et al. Synovial sarcoma of nerve. Hum Pathol 2011;42:568-577.

15 Hruban RH, Shiu MH, Senie RT, et al. Malignant peripheral nerve sheath tumors of the buttock and lower extremity. A study of 43 cases. Cancer 1990;66: 1253-1265.

16 Brems H, Beert E, de Ravel T, et al. Mechanisms in the pathogenesis of malignant tumours in neurofibromatosis type 1. Lancet Oncol 2009;10:508-515.

17 Turc-Carel C, Dal Cin P, Limon J, et al. 18 in synovial sarcoma. Cancer Genet Cytogenet 1986;23:93.

18 Britsch S, Goerich DE, Riethmacher D, et al. The transcription factor Sox10 is a key regulator of peripheral glial development. Genes Dev 2001;15:66-78.

19 Kuhlbrodt K, Herbarth B, Sock E, et al. Sox10, a novel transcriptional modulator in glial cells. J Neurosci 1998;18:237-250.

20 Palla B, Su A, Binder S, et al. SOX10 expression distinguishes desmoplastic melanoma from its histologic mimics. Am J Dermatopathol 2013;35:576-581.

21 Nonaka D, Chiriboga L, Rubin BP. Sox10: a panSchwannian and melanocytic marker. Am J Surg Pathol 2008;32:1291-1298.

22 Karamchandani JR, Nielsen TO, van de Rijn M, et al. Sox10 and S100 in the diagnosis of soft-tissue neoplasms. Appl Immunohistochem Mol Morphol 2012;20:445-450.

23 Cimino-Mathews A, Subhawong AP, Elwood H, et al. Neural crest transcription factor Sox10 is preferentially expressed in triple-negative and metaplastic breast carcinomas. Hum Pathol 2012;44:959-965.

24 Wong WW, Hirose T, Scheithauer BW, et al. Malignant peripheral nerve sheath tumor: analysis of treatment outcome. Int J Radiat Oncol Biol Phys 1998;42: 351-360.

25 Horvai AE, Kramer MJ, O’Donnell R. Beta-catenin nuclear expression correlates with cyclin D1 expression in primary and metastatic synovial sarcoma: a tissue microarray study. Arch Pathol Lab Med 2006;130: 792-798.

26 Rubin BP, Fletcher CD, Inwards C, et al. Protocol for the examination of specimens from patients with soft tissue tumors of intermediate malignant potential, malignant soft tissue tumors, and benign/locally aggressive and malignant bone tumors. Arch Pathol Lab Med 2006;130:1616-1629.

27 Adams EJ, Green JA, Clark AH, et al. Comparison of different scoring systems for immunohistochemical staining. J Clin Pathol 1999;52:75-77.

28 Horvai AE, DeVries S, Roy $\mathrm{R}$, et al. Similarity in genetic alterations between paired well-differentiated and dedifferentiated components of dedifferentiated liposarcoma. Mod Pathol 2009;22:1477-1488.

29 Hirakawa N, Naka T, Yamamoto I, et al. Overexpression of bcl-2 protein in synovial sarcoma: a comparative study of other soft tissue spindle cell sarcomas and an additional analysis by fluorescence in situ hybridization. Hum Pathol 1996;27:1060-1065.

30 Shimada S, Tsuzuki T, Kuroda M, et al. Nestin expression as a new marker in malignant peripheral nerve sheath tumors. Pathol Int 2007;57:60-67.

31 Suster S, Fisher C, Moran CA. Expression of bcl-2 oncoprotein in benign and malignant spindle cell tumors of soft tissue, skin, serosal surfaces, and gastrointestinal tract. Am J Surg Pathol 1998;22: 863-872.

32 Foo WC, Cruise MW, Wick MR, et al. Immunohistochemical staining for TLE1 distinguishes synovial sarcoma from histologic mimics. Am J Clin Pathol 2011;135:839-844.

33 Knosel T, Heretsch S, Altendorf-Hofmann A, et al. TLE1 is a robust diagnostic biomarker for synovial sarcomas and correlates with $\mathrm{t}(\mathrm{X} ; 18)$ : analysis of 319 cases. Eur J Cancer 2010;46:1170-1176.

34 Kosemehmetoglu K, Vrana JA, Folpe AL. TLE1 expression is not specific for synovial sarcoma: a whole section study of 163 soft tissue and bone neoplasms. Mod Pathol 2009;22:872-878.

35 Terry J, Saito T, Subramanian S, et al. TLE1 as a diagnostic immunohistochemical marker for synovial sarcoma emerging from gene expression profiling studies. Am J Surg Pathol 2007;31:240-246.

36 O’Sullivan MJ, Kyriakos M, Zhu X, et al. Malignant peripheral nerve sheath tumors with $t(X ; 18)$. A pathologic and molecular genetic study. Mod Pathol 2000;13:1336-1346.

37 Ladanyi M, Woodruff JM, Scheithauer BW, et al. Re: O’Sullivan MJ, Kyriakos M, Zhu X, Wick MR, Swanson PE, Dehner LP, Humphrey PA, Pfeifer JD. Malignant peripheral nerve sheath tumors with $t(X ; 18)$. A pathologic and molecular genetic study. Mod pathol 2000;13:1336-46. Mod Pathol 2001;14:733-737.

38 Gubbay J, Collignon J, Koopman P, et al. A gene mapping to the sex-determining region of the mouse $\mathrm{Y}$ chromosome is a member of a novel family of embryonically expressed genes. Nature 1990;346: $245-250$.

39 Pevny LH, Lovell-Badge R. Sox genes find their feet. Curr Opin Genet Dev 1997;7:338-344.

40 Wegner M, Stolt CC. From stem cells to neurons and glia: a Soxist's view of neural development. Trends Neurosci 2005;28:583-588.

41 Wegner M. From head to toes: the multiple facets of Sox proteins. Nucleic Acids Res 1999;27:1409-1420.

42 Koopman P, Schepers G, Brenner S, et al. Origin and diversity of the SOX transcription factor gene family: genome-wide analysis in Fugu rubripes. Gene 2004; 328:177-186.

43 Kordes U, Cheng YC, Scotting PJ. Sox group E gene expression distinguishes different types and maturational stages of glial cells in developing chick and mouse. Brain Res Dev Brain Res 2005;157: 209-213.

44 Pytel P, Karrison T, Can G, et al. Neoplasms with Schwannian differentiation express transcription factors known to regulate normal schwann cell development. Int J Surg Pathol 2012;18:449-457. 